\title{
Simulation of Recrystallization in Iron Single Crystals
}

\author{
Tatiana Caneda Salazar, Weslley Luiz da Silva Assis, Paulo Rangel Rios* \\ Escola de Engenharia Industrial Metalúrgica de Volta Redonda, \\ Universidade Federal Fluminense - UFF, \\ Av. dos Trabalhadores, 420, 27255-125 Volta Redonda - RJ, Brazil
}

Received: January 7, 2008; Revised: February 26, 2008

\begin{abstract}
Recrystallization of an iron single crystal was reported in detail by Vandermeer and Rath (V\&R). We present predictions of recrystallization based on cellular automata (CA) simulations, and compare them with the data and analysis from V\&R's study. Agreement is found between our CA simulations and V\&R's results, provided that the CA simulations were carried out using a sufficient dynamic range for time, precision spatial dimensionalization, and accommodation of grain shape effects inherent in CA techniques.
\end{abstract}

Keywords: microstructure, kinetics, recrystallization, phase transformations, computer simulation, cellular automata

\section{Introduction}

Recrystallization ${ }^{1}$ can be grouped among 'nucleation and growth' reactions, but it remains kinetically distinct from other well-studied nucleation and growth reactions, such as precipitation. Firstly, the nucleation mechanism associated with recrystallization cannot normally be described by classical fluctuation-based nucleation theory. Nucleation sites are usually determined by microstructural features that are often already present or forming early in the reaction. Thus, nucleation frequently takes place quickly and preferred sites become saturated early in the reaction, leading to the well-known 'site-saturated' situation. Site-saturation, for example, was recently shown to take place in recrystallizing aluminum by $3 \mathrm{XRD}$ techniques ${ }^{2}$. An additional important point is that knowledge of the deformed state holds a place of paramount importance in recrystallization. In the deformed state a heterogeneous distribution of stored energy and of grain orientation, i.e., crystallographic texture, may be present. Furthermore, interface migration during recrystallization is considered to be a local phenomenon, in the sense that migration depends only on the local driving force, which is the difference in chemical potential between the growing grain and the matrix adjacent to the moving interface, and, of course, on the interface mobility. Solute transport is thought not to occur, as it usually does in precipitation reactions, although the influence of solutes on interface mobility, might be important. The large number of studies on this complex phenomenon is justified not only by its intrinsic scientific interest, but also by its impact on the industrial processing of metallic materials ${ }^{1}$.

Nowadays, recrystallization may be qualitatively interpreted and quantitatively modeled with the help of both analytical models and computer simulations. These methodologies are not mutually exclusive but may be combined effectively for a better understanding of the phenomenon. Analytical models often provide a preliminary idea of the general trends and order of magnitude of relevant parameters that could prove useful in the design of the computer simulation, even in situations in which their assumptions might not be strictly valid. We shall compare CA simulation in this paper to experimental data and analytical modeling obtained by Vandermeer and Rath ${ }^{3}$ (V\&R) in their study of the recrystallization of an iron single crystal. Their study produced one of the best published datasets on recrystallization kinetics. The absence of interfering grain boundaries in their single crystal specimens, achieving a uniform distribution of the nuclei that led to equiaxed growth, combine to make this system simpler to analyze than would specimens of polycrystalline metals. Moreover, V\&R executed a thorough and careful quantitative analytical treatment (see description below) of their data that can be compared with our CA simulation results.

The current analytical methodology employed to model recrystallization is based on the early work of Johnson-Mehl ${ }^{4}$, Avrami ${ }^{5-7}$ and Kolmogorov $^{8}$ (JMAK). JMAK uses a single microstructural descriptor, i.e., the volume fraction of transformed regions, $\mathrm{V}_{\mathrm{V}^{*}}$ Moreover, JMAK's theory was subsequently extended by DeHoff and Gokhale ${ }^{9-12}$, who proposed the use of an additional microstructural measure, namely, the interfacial area per unit of volume between transformed and untransformed regions, $S_{\mathrm{v}}$, and the associated kinetic concept of microstructural path. Vandermeer and coworkers ${ }^{13}$ extended that concept in a theoretical treatment covering variable nucleation and growth rates, as well as inclusion of non-spherical regions. They called their extended analysis the microstructural path method (MPM).

The MPM analyses recrystallization in an 'extended space'. The key quantities introduced in MPM are the extended volume fraction, $\mathrm{V}_{\mathrm{VE}}$, and the extended interface area per unit of volume between transformed grains and untransformed regions, $\mathrm{S}_{\mathrm{VE}}$. The former is the sum of the volumes of all growing regions, whereas the latter is the sum of the interface area of all individual grains. Extended quantities are calculated by assuming that the extended regions grow freely, that is, that they grow as if each were the only regions growing, with any region extending its size indefinitely without impinging on any other region. The extended quantities can be transformed into measurable quantities of the real microstructure by means of the fundamental relationships

$$
\mathrm{dV}_{\mathrm{vE}}=\frac{\mathrm{dV}_{\mathrm{v}}}{1-\mathrm{V}_{\mathrm{v}}}
$$

which in integrated form is

$$
\mathrm{V}_{\mathrm{V}}=1-\exp \left(-\mathrm{V}_{\mathrm{E}}\right)
$$

*e-mail: prrios@metal.eeimvr.uff.br 


$$
\mathrm{S}_{\mathrm{VE}}=\frac{\mathrm{S}_{\mathrm{V}}}{1-\mathrm{V}_{\mathrm{V}}}
$$

The advantage of this methodology is that the extended quantities derive from experimental measurements of just $\mathrm{V}_{\mathrm{v}}$ and $\mathrm{S}_{\mathrm{v}}$, both of which may be determined from planar sections using standard stereological techniques.

Equations 1 and 2 are mathematically exact, but their derivation involves the underlying assumption that nuclei are located at random in the matrix. Recently, it has become increasingly clear that in many reactions the nuclei are not located at random. Thus, additional parameters that test for non-randomness have been advocated.

Vandermeer ${ }^{14}$ proposed in a recent paper that the so-called contiguity ratio, $\mathrm{C}_{\beta}$, should be included in the MPM, owing to its sensitivity to deviations from randomness in nuclei location. The contiguity function requires measurement of the interface area per unit of volume between the transformed phase, $\beta$, and untransformed regions, $\alpha$. Here, specifically, the interphase interfacial area density is designated by the symbol $\mathrm{S}_{\mathrm{v} \beta}$,

$$
\mathrm{C}_{\beta}=\frac{2 \mathrm{~S}_{\mathrm{v} \beta}}{\mathrm{S}_{\mathrm{v}}+2 \mathrm{~S}_{\mathrm{v} \beta}}
$$

An alternative approach proposed recently by Rios et al. ${ }^{15}$ defines a new function called the impingement function, $\phi\left(V_{v}\right)$, to replace the KJMA impingement factor, $1-\mathrm{V}_{\mathrm{v}}$, when nuclei are located nonrandomly through the matrix. The function $\phi$, itself, is a measure of the non-randomness or underlying 'signature' of nuclei located at nonrandom sites. Full details are given in Rios et al. ${ }^{15}$. Equations 1 and 2 may now be rewritten by substituting $\phi\left(\mathrm{V}_{\mathrm{v}}\right)$,

$$
\begin{aligned}
& \mathrm{dV}_{\mathrm{E}}=\frac{\mathrm{dV}_{\mathrm{V}}}{\phi\left(\mathrm{V}_{\mathrm{V}}\right)} \\
& \mathrm{S}_{\mathrm{E}}=\frac{\mathrm{S}_{\mathrm{V}}}{\phi\left(\mathrm{V}_{\mathrm{V}}\right)}
\end{aligned}
$$

Unfortunately, earlier studies of recrystallization kinetics did not report these parameters, often using JMAK equations in the form

$$
\mathrm{V}_{\mathrm{v}}=1-\exp \left(-\mathrm{kt}^{\mathrm{n}}\right)
$$

where $\mathrm{n}$ is the time exponent, and $\mathrm{K}$ depends on the temperature. Notice that, comparing Equation 6 with Equation 1 gives $\mathrm{V}_{\mathrm{VE}}=\mathrm{Kt}^{\mathrm{n}}$. In addition to Equation 6 the microstructural path function, $\mathrm{S}_{\mathrm{v}}\left(\mathrm{V}_{\mathrm{v}}\right)$ or $\mathrm{S}_{\mathrm{VE}}\left(\mathrm{V}_{\mathrm{VE}}\right)$, is also used. Details of the path functions will be provided in the sections below.

However useful the analytical approach might be, it suffers from some shortcomings. One example, as already pointed out, occurs when the nuclei are not randomly located, in which case the two basic analytical equations, Equations 1 and 2, become invalid. The availability of computer simulations of microstructural evolution adds quantitative information into the complexity of microstructures that extends our insights beyond that of analytical methods. Actually, as we advocate here, it is interesting, whenever possible, to couple both techniques. Among the wide range of simulation techniques now available, an especially convenient simulation technique applicable to recrystallization is that of $\mathrm{CA}$, as shown in the pioneering work of Hesselbarth and Göbel ${ }^{16}$. Therefore, it is not surprising that CA is the choice of many researchers simulating recrystallization, including Gottstein ${ }^{17,18}$ and Raabe ${ }^{19,20}$ and many others ${ }^{21-26}$. We too have published a series of papers using CA simulations ${ }^{15,27-31}$. In those papers CA simulation was shown to be geometrically sound in 2- $\mathrm{d}^{28}$ and $3-\mathrm{d}^{30}$ by comparing the results with exact analytical theory available for recrystallization of randomly located nuclei. Moreover, we also used CA simulations to study the influence of non-randomness of nuclei distribution ${ }^{15,27,29,31}$. Nevertheless, our studies lacked direct comparison of CA simulation to experimental data, as is provided here. We choose as 'simple' experimental realization of recrystallization the single crystal system investigated by $V \& R^{3}$.

\section{Cellular Automata in 3d}

The implementation of CA simulation follows that of Hesselbarth and Göbe ${ }^{16}$ by using the von Neumann neighborhood criterion in 3-d. The matrix consisted of a cubic lattice with $300 \times 300 \times 300$ cells. The interface velocity used depends on time, the implementation of which is describe in detail in Assis ${ }^{32}$. A probabilistic approach was used to implement the variable velocity. For a constant interface velocity, whenever a certain cell had one of its neighbors transformed it would itself transform. In the probabilistic approach a probability of transformation is assigned to this cell. Of course this probability of transformation is equal to one if the velocity is constant. A probability of transformation that decreases with time results in an interface velocity that decreases with reaction time. One of the problems with the probabilistic approach is that it may lead to a change of shape when the growth of an isolated grain is considered. This change of shape during the reaction may cause erroneous microstructural path results ${ }^{32}$. In the present simulation this was avoided by using an algorithm specially developed by Assis ${ }^{32}$ to keep the shape of the transformed regions in extended space constant even though the approach remained probabilistic. The velocity was set to decrease as a function of (time) $)^{-0.4}$, which is the same time dependence found by V\&R. Comparison of their data with our CA simulation requires assigning units both for the cell edge and the time step. The cell edge was chosen to be $1.5 \mu \mathrm{m}$ in length. This choice was made to insure that a reasonable number of nuclei would be included in the simulation. Specifically, 220 nuclei is the number of nuclei per unit of volume found by $V \& R$, or $2.4 \cdot 10^{-6}$ nuclei. $\mu \mathrm{m}^{-3}$. A smaller edge length would have included too few nuclei that might have caused problems with the statistical precision of the simulation. Prior experience with CA simulations show that it is important to include at least 100 nuclei in the simulation to give a reliable statistics. The nucleation was sitesaturated, i.e., all nuclei appeared instantaneously at $t=0$, as was also assumed in the analytical treatment of V\&R. The correlation between the cellular automata time step, $\mathrm{t}_{\mathrm{CA}}$, and V\&R's dimensionless time is detailed in a later section.

\section{Data and Analytics from V\&R}

V\&R investigated the recrystallization of an iron single crystal, cold rolled $70 \%$ over a temperature range of $450-600{ }^{\circ} \mathrm{C}$. Their data, as depicted in the figures contained in their paper were digitalized for comparison with our simulations. V\&R were able to normalize all data over the experimental temperature range by means of a dimensionless time, $\tau$. Their equations for extended volume fraction and interfacial area as functions of $\tau$ are

$$
\begin{aligned}
& \mathrm{V}_{\mathrm{VE}}=\mathrm{B}_{\mathrm{n}} \cdot \tau^{\mathrm{n}} \\
& \mathrm{S}_{\mathrm{VE}}=\mathrm{K}_{\mathrm{m}} \cdot \tau^{\mathrm{m}}
\end{aligned}
$$

where $\tau$ is scaled time defined as

$$
\tau_{\mathrm{i}}=\frac{\mathrm{t}}{\mathrm{t}_{\mathrm{i}}} \exp \left(-\frac{\mathrm{Q}}{\mathrm{RT}}\right)
$$

Here $\mathrm{t}$ is time in minutes; $\mathrm{t}_{\mathrm{i}}$ is a constant; and $\mathrm{i}=\mathrm{v}, \mathrm{s}$ denotes either volume fraction or interfacial area per unit of volume, respec- 
tively. Also, $\mathrm{t}_{\mathrm{v}}=1.836 \cdot 10^{-20} \mathrm{~s}$ and $\mathrm{t}_{\mathrm{s}}=1.614 \cdot 10^{-20} \mathrm{~s} ; \mathrm{Q}=335 \mathrm{~kJ} \cdot \mathrm{mol}^{-1}$; $\mathrm{n}=1.9 ; \mathrm{m}=1.28 ; \mathrm{B}_{\mathrm{n}}=0.118, \mathrm{~K}_{\mathrm{m}}=0.0157 \mu \mathrm{m}^{-1} ; \mathrm{R}=8.3144 \mathrm{~J} \cdot \mathrm{mol}^{-1}$. Thus, either a $\tau_{\mathrm{v}}$ or $\tau_{\mathrm{s}}$ was selected by $\mathrm{V} \& \mathrm{R}$, depending whether the normalized time refers to $\mathrm{V}_{\mathrm{VE}}$ or $\mathrm{S}_{\mathrm{VE}}$, respectively.

The CA time step will be related to $\tau_{v}$. For the present purposes it is more convenient to choose a single dimensionless time, and $\tau=\tau$, was selected. Thus, $\tau_{\mathrm{v}}$ and $\tau_{\mathrm{s}}$ are replaced by $\tau$ in what follows.

In order to maintain consistency, the experimental data for $S_{\mathrm{VE}}$ vs. $\tau_{\mathrm{s}}$ had to be corrected. This correction consisted in multiplying $\tau_{\mathrm{s}}$ (experimental) by $\mathrm{t}_{\mathrm{s}} / \mathrm{t}_{\mathrm{v}}$, and $\mathrm{S}_{\mathrm{VE}}$ (experimental) by $\left(\mathrm{t}_{\mathrm{s}} / \mathrm{t}_{\mathrm{v}}\right)^{1.28}$. This procedure insures that Equation 8 retains the same form and $\mathrm{K}_{\mathrm{m}}$ value does not change when $\tau_{\mathrm{s}}$ is replaced by $\tau$. Obviously, no correction was required for $V \& R$ 's experimental datasets for $S_{\mathrm{VE}} \mathrm{VS}$. $\mathrm{V}_{\mathrm{VE}}$. The reason why $V \& R$ used two distinct dimensionless times was that they adopted $\mathrm{V}_{\mathrm{V}}=0.1$ and $\mathrm{S}_{\mathrm{V}}=0.010 \mu \mathrm{m}^{-1}$ as their reference values, as this facilitated the determination of their reference times at each temperature. This choice resulted in different reference times. The present approach — making the reference times equal — implicitly uses $\mathrm{S}_{\mathrm{v}}=0.014 \mu \mathrm{m}^{-1}$ as the reference value.

\section{Results}

The 3-d microstructure generated by the simulations is illustrated in Figures 1a-e, for increasing volume fractions transformed: 1a) Nucleation: $\left.\left.\left.\mathrm{V}_{\mathrm{v}} \approx 0 ; 1 \mathrm{~b}\right) \mathrm{V}_{\mathrm{v}}=0.15 ; 1 \mathrm{c}\right) \mathrm{V}_{\mathrm{V}}=0.30 ; 1 \mathrm{~d}\right) \mathrm{V}_{\mathrm{v}}=0.50$; and $1 \mathrm{e}$ ) $\mathrm{V}_{\mathrm{V}}=1$. The sequence of figures clearly shows the progression of the impingement. Notice that at $\mathrm{V}_{\mathrm{v}}=0.5, \mathrm{~S}_{\mathrm{v}}$ becomes a maximum (see Figures 4 and 8) Therefore, in spite of impingement, $S_{v}$ increases up to $\mathrm{V}_{\mathrm{V}}=0.5$, when impingement starts to predominate and $\mathrm{S}_{\mathrm{V}}$ decreases until it reaches zero when $\mathrm{V}_{\mathrm{v}}=1$.

A key issue when comparing CA simulation with V\&R's analytical treatment is the difference in the shape of the growing grain. Traditional MPM formulations assume that the grains are spherical, and inspection of V\&R's micrograph of the early stages of transformation indeed suggests that a spherical grain approximation is reasonable. CA simulated grains are, however, not spherical, as may be seen in Figure 1. The non-sphericity occurs because CA grains are modeled initially from cubic mesh cells, and so their interfaces are never smooth. Of course, as the grains 'collide' with one another during impingement their shapes will change in real space. Insofar as we are dealing with 'extended' quantities, it is important to insure that the grain shapes in extended space, where the grains grow without impingement, remain the same.

It is well-known that for a given volume a spherical grain exhibits the minimum surface area. Thus, the surface area of CA grains is always larger than that of spherical grains of equal volume. As a consequence, direct quantitative comparison of the interfacial area generated by $\mathrm{CA}$ simulation with the analytical model becomes possible only by multiplying by a suitable shape factor. In the CA implementation used here, care was taken to insure that the shape of the growing grains in extended space remained constant. As a

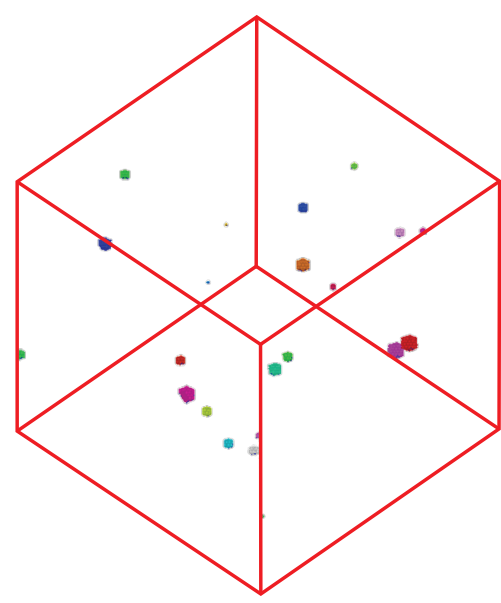

(a)

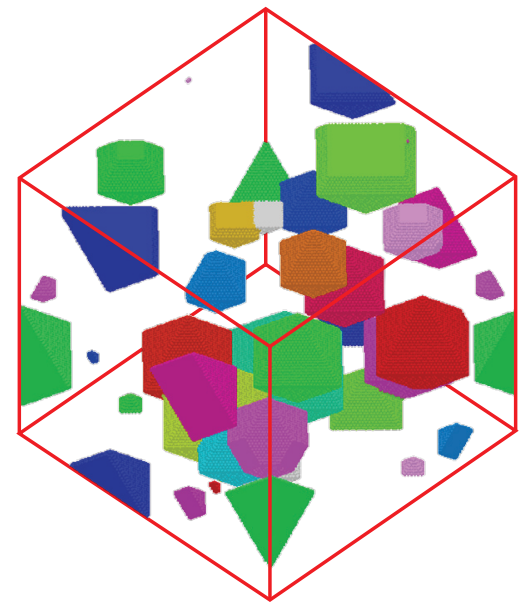

(b)

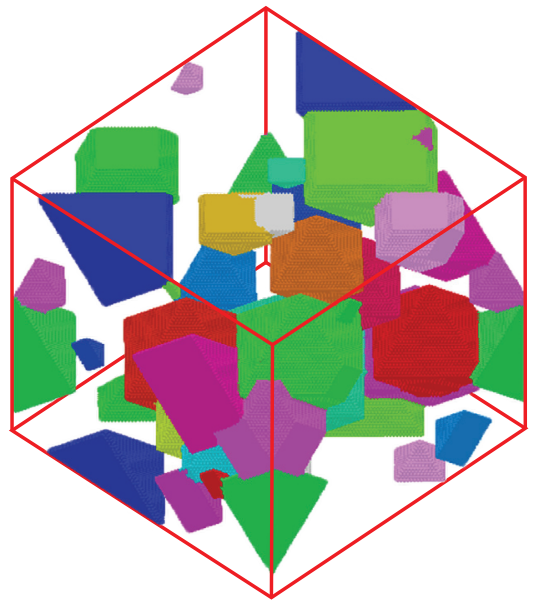

(c)

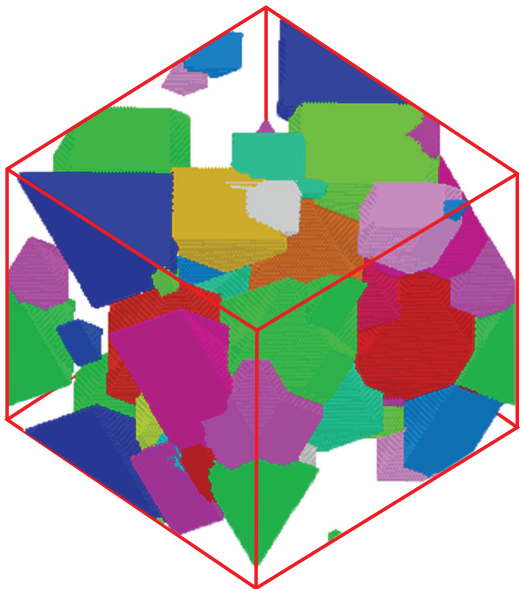

(d)

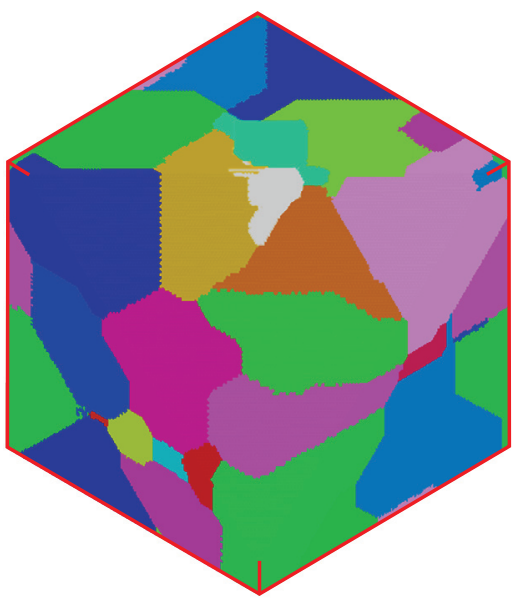

(e)

Figure 1. CA simulated microstructures of the recrystallization of an iron single crystal studied by Vandermeer and Rath ${ }^{3}$. The increase in the fraction recrystallized, $\mathrm{V}_{\mathrm{v}}$, is shown: a) Nucleation: $\mathrm{V}_{\mathrm{v}} \approx 0$; b) $\mathrm{V}_{\mathrm{v}}=0.15$; c) $\mathrm{V}_{\mathrm{v}}=0.30 ;$ d) $\mathrm{V}_{\mathrm{v}}=0.50$; and e) $\mathrm{V}_{\mathrm{v}}=1$. 
consequence, their shape is independent of time. Interestingly, this important, but non-obvious point is seldom ever mentioned in published CA simulations. An in-depth investigation of CA grain shape in 2-d and 3-d may be found in Rios et al. ${ }^{28,30}$.

The theoretical microstructural path in extended space is given by

$$
\mathrm{S}_{\mathrm{VE}}=\mathrm{C}\left(\mathrm{V}_{\mathrm{vE}}\right)^{\mathrm{q}}
$$

where $\mathrm{q}=2 / 3$ for site-saturated reactions.

For CA simulations, $\mathrm{C}_{\mathrm{CA}}=3 \cdot\left(36 \mathrm{~N}_{\mathrm{v}}\right)^{\frac{1}{3}}$ and for a spherical model $\mathrm{C}_{\mathrm{sp}}=\left(36 \pi \mathrm{N}_{\mathrm{v}}\right)^{\frac{1}{3}}$. Thus, the ratio of these factors is

$$
\frac{\mathrm{C}_{\mathrm{CA}}}{\mathrm{C}_{\mathrm{sp}}}=\frac{3}{\pi^{1 / 3}} \approx 2.048
$$

If one assumes that the extended volume is identical in both cases, the relationship between extended interfacial areas becomes

$$
\frac{\mathrm{S}_{\mathrm{VE}}^{\mathrm{CA}}}{\mathrm{S}_{\mathrm{VE}}^{\mathrm{sp}}}=\frac{3}{\pi^{1 / 3}} \approx 2.048
$$

where $\mathrm{S}_{\mathrm{VE}}^{\mathrm{CA}}$ and $\mathrm{S}_{\mathrm{VE}}^{\mathrm{sp}}$ are the extended interfacial area per unit of volume of the CA simulation and of the spherical model, respectively. Therefore, in all comparisons that follow, extended interfacial area generated by CA simulation will always be divided by 2.048 . Bearing this in mind, all extended interfacial areas will be designated simply by $\mathrm{S}_{\mathrm{VE}}$.

Figure 2 shows the microstructural path data in extended space in a $\log _{10}-\log _{10}$ plot. V\&R fitted a straight line to their experimental data. Their best-fit yielded $\mathrm{C}=0.0650 \mu \mathrm{m}^{-1}$ and $\mathrm{q}=0.64$. CA simulation data are also depicted in Figure 2, and show good agreement with the experimental data. $V \& R$ also calculated the number of grains per unit of volume by setting $C=0.065=\left(36 \pi \mathrm{N}_{\mathrm{V}}\right)^{1 / 3}$ and obtained $2.4 \cdot 10^{-6}$ nuclei. $\mu \mathrm{m}^{-3}$. This value was used in our CA simulation as already mentioned in section 2 .

In order to assess the scatter of the data, the microstructural path is plotted in real space in Figure 3. The solid line in Figure 3 corresponds to the best-fitted line by V\&R in Figure 2. Their experimental data lie somewhat above the best-fitted line and the simulation for the mid-range of $\mathrm{V}_{\mathrm{v}^{*}}$. Still the agreement remains reasonable. For further comparison, the line corresponding to the exact microstructural path

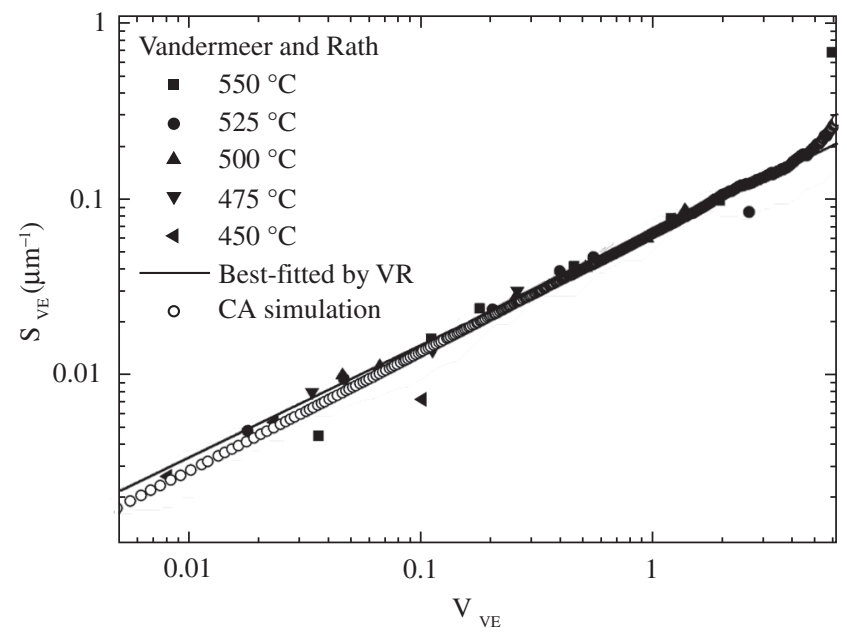

Figure 2. Microstructural path in extended space: extended interfacial area per unit of volume between transformed and untransformed region, $\mathrm{S}_{\mathrm{VE}}$, plotted against extended volume fraction transformed, $\mathrm{V}_{\mathrm{VE}}$. for spherical grains with $\mathrm{C}=0.065$ and $\mathrm{q}=2 / 3$ is also shown. All three approaches, best-fitted, exact, and CA, in fact, fall close to one another and give a reasonable description of the experimental data, despite that these data are unavoidably subjected to experimental errors. Unfortunately, V\&R did not report the magnitude of these errors. We note that for site-saturated recrystallization the microstructural path does not depend on the interface velocity. Thus, for Figures 2 and 3 there was no need to relate the CA time step and the experimental time, as will be done next.

In order to compare $V \& R$ results with the present $C A$ simulation, V\&R's dimensionless time was used. A $\log _{10}-\log _{10}$ plot of $\mathrm{V}_{\mathrm{VE}}$ vs. $\tau$ is shown in Figure 4. The method by which the CA time step, $\mathrm{t}_{\mathrm{CA}}$, was related to $\mathrm{V} \& \mathrm{R}$ 's dimensionless time, $\tau$, is straightforward. For $\tau=1$ and $\tau=8$ the corresponding CA time steps, $\mathrm{t}_{\mathrm{CA}}(\tau=1)$ and $\mathrm{t}_{\mathrm{CA}}(\tau=8)$ were found that gave the same value of $\mathrm{V}_{\mathrm{VE}}$. For example, $\mathrm{V}_{\mathrm{VE}}(\tau=1)=\mathrm{V}_{\mathrm{VE}}\left(\mathrm{t}_{\mathrm{CA}}(\tau=1)\right)$. A relationship between $\tau$ and $\mathrm{t}_{\mathrm{CA}}$ was then found from the ratio of both intervals

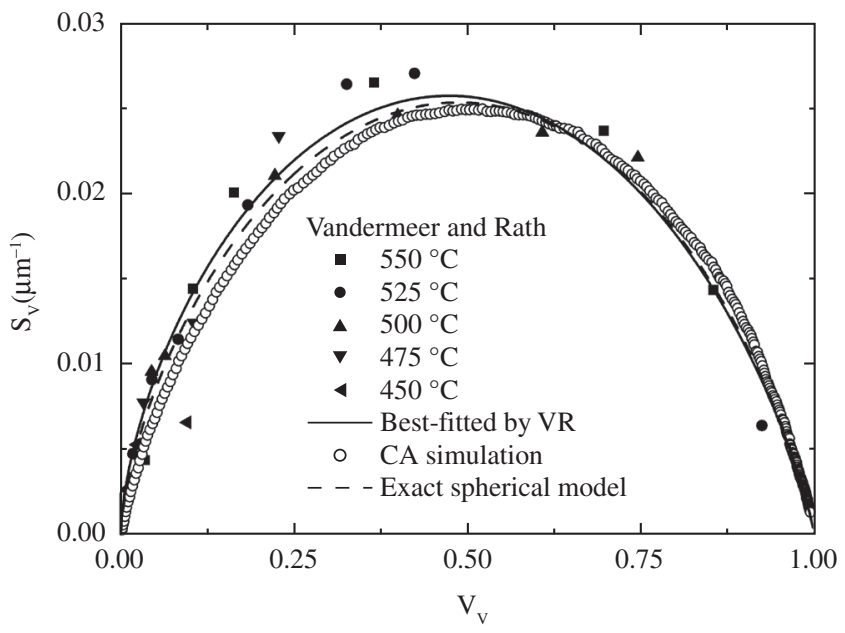

Figure 3. Microstructural path in real space: interfacial area per unit of volume between transformed and untransformed region, $S_{\mathrm{v}}$, plotted against volume fraction transformed, $\mathrm{V}_{\mathrm{v}}$.

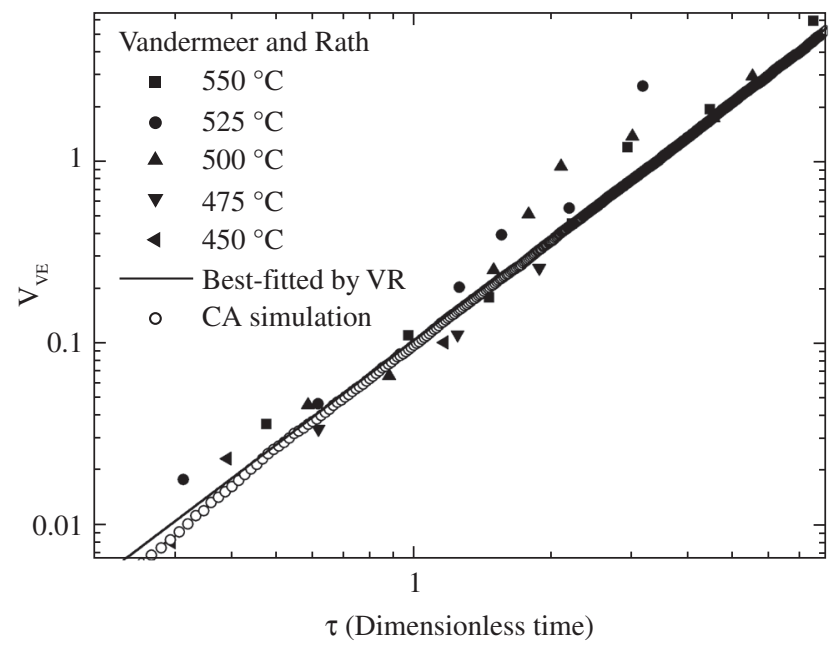

Figure 4. Extended volume fraction transformed, $\mathrm{V}_{\mathrm{VE}}$, as a function of dimensionless time, $\tau$, for $\mathrm{V} \& \mathrm{R}$ 's recrystallization of an iron single crystal and for CA simulation. 


$$
\tau=\left(\frac{8-1}{t_{\mathrm{CA}}(\tau=8)-\mathrm{t}_{\mathrm{CA}}(\tau=1)}\right) \mathrm{t}_{\mathrm{CA}} \cong \frac{\mathrm{t}_{\mathrm{CA}}}{81.7}
$$

Figure 4 shows that there is agreement between the CA simulation and V\&R's data. The solid line in Figure 4 corresponds to V\&R's best-fitted straight line to their data, which gives Equation 7 in section 3 .

Figure 5 provides a better idea of the data scatter by re-plotting Figure 4 in real space. The data corresponding to the mid-range times, $\tau \approx 3$, seems to be somewhat farther from the fitted line. Again, CA simulation and best-fitted line remain close. Note, that the solid line in Figure 5 corresponds to the solid line best-fitted by V\&R in Figure 4.

A $\log _{10}-\log _{10}$ plot of $S_{\mathrm{VE}}$ vs. $\tau$ is shown in Figure 6. It is clear that good agreement occurs between CA simulation and V\&R's data. The solid line in Figure 6 corresponds to V\&R's best-fitted straight line to the data, resulting in Equation 8 in section 3. Figure 7 is a plot of Figure 6 in real space. The data up to $\tau \approx 3$ seems to give better agreement than that at later times. Again, CA simulation and the best-fitted

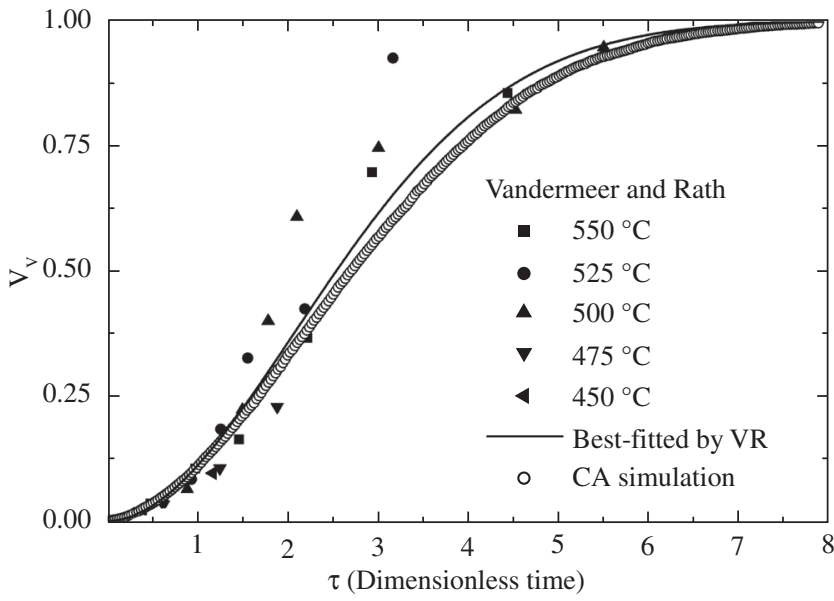

Figure 5. Volume fraction transformed, $\mathrm{V}_{\mathrm{v}}$, as a function of dimensionless time, $\tau$, for Vandermeer and Rath's recrystallization of an iron single crystal and for CA simulation.

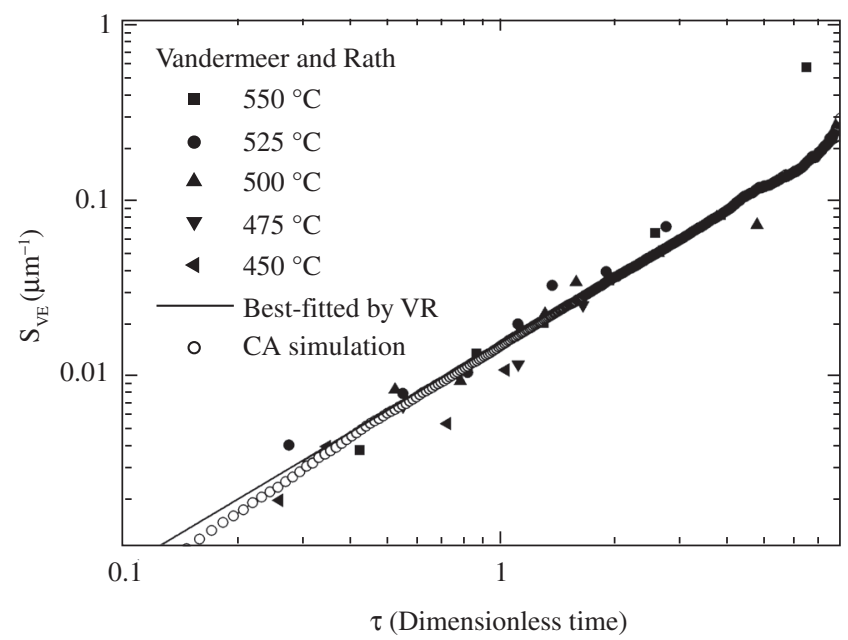

Figure 6. Extended interfacial area per unit of volume between transformed and untransformed regions, $\mathrm{S}_{\mathrm{VE}}$, plotted as a function of dimensionless time, $\tau$, for V\&R's recrystallization of an iron single crystal and for CA simulation. line remain close. We note that the solid line in Figure 6 corresponds to the solid line best-fitted by V\&R in Figure 7.

The grain boundary velocity, $\mathrm{G}$, can be calculated by means of the Cahn and Hagel ${ }^{33}$ equation

$$
\mathrm{G}=\frac{1}{\mathrm{~S}_{\mathrm{VE}}} \frac{\mathrm{dV}_{\mathrm{VE}}}{\mathrm{dt}}=\frac{1}{\mathrm{~S}_{\mathrm{V}}} \frac{\mathrm{dV}_{\mathrm{V}}}{\mathrm{dt}}
$$

Inserting $\mathrm{V}_{\mathrm{VE}}$ and $\mathrm{S}_{\mathrm{VE}}$ vs. $\tau$ into the Cahn and Hagel equation using the $\mathrm{V} \& \mathrm{R}$ data, Equations 7 and 8, gives

$$
\mathrm{G}_{\mathrm{VR}}=14.4 \tau^{-0.38} \mu \mathrm{m} / \text { dimensionless time }
$$

For the CA simulations, the velocity was evaluated numerically from the simulated data, to which an analytical expression was best-fitted, as shown in Figure 8. That procedure yields

$\mathrm{G}_{\mathrm{CA}}=14.3 \tau^{-0.4} \mu \mathrm{m} /$ dimensionless time

where the correlation coefficient $\mathrm{R}=0.97$.

$\mathrm{G}_{\mathrm{CA}}$ and $\mathrm{G}_{\mathrm{VR}}$ are indeed close to one another. Both are shown in Figure 8 and appear almost indistinguishable in this plot. In

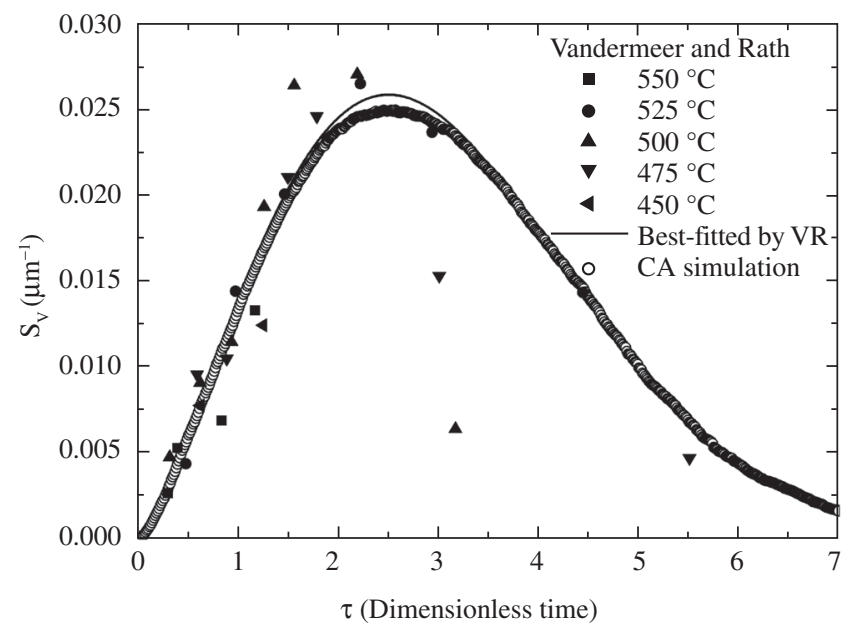

Figure 7. Interfacial area per unit of volume between transformed and untransformed regions, $S_{v}$, plotted as a function of dimensionless time, $\tau$, for $V \& R$ 's recrystallization of an iron single crystal and for CA simulation.

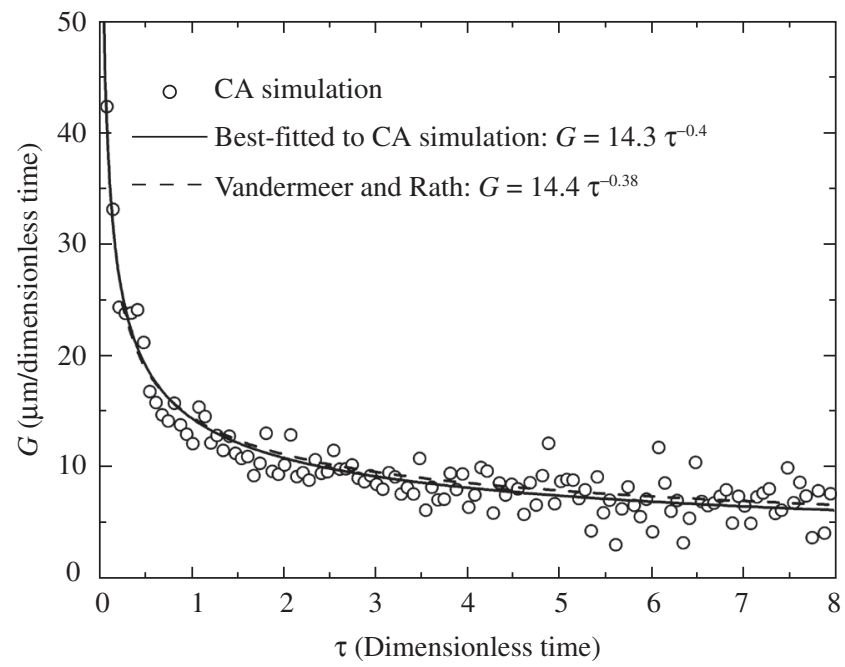

Figure 8. Grain boundary velocity, $G$, as a function of dimensionless time, $\tau$, for V\&R's recrystallization of an iron single crystal and for CA simulation. 
order to improve visualization not all simulated points are plotted in Figure 8.

\section{Discussion}

Overall, good agreement was obtained between CA simulations with experimental recrystallization data as well as with V\&R's analytical description of their data. The plots of real, rather than extended, quantities indicate that the scatter of these data was significant. Some departures from the experimental data were apparent in all plots of real quantities, with perhaps the best agreement exhibited by the microstructural path, $\mathrm{S}_{\mathrm{v}}$ vs. $\mathrm{V}_{\mathrm{v}}$, plot. In all three real quantity plots, viz., Figures 2, 4 and 6, deviations of experimental data from theory appear, but these deviations did not occur at quite the same point in the reaction evolution. The causes of these disparities are difficult to determine in view of the scatter in the original data. The scatter itself could provide the reason behind the disparity. Another possibility would be non-randomness. However, if this non-randomness is weak, and falls below the experimental limits of error, then one might still consider the nucleation sites to be 'randomly' located.

Another issue might be the differences in the shapes of CA, real, and spherical grains. Such differences are less problematic if accounted for by a shape factor that is independent of time, as was done here. Clearly, the correction factor used here allowed the quantitative data to match, but still one must bear in mind that the simulated microstructures do have different interfacial area per unit of volume than the real ones. Therefore, considerable care must be exercised when comparing absolute values of interfacial areas of simulated and real microstructures. This is especially the case when modeling complex microstructures involving deformation gradients and textures. These cases are beyond theoretical analysis precluding some type of shape factor correction. Even accounting for shape differences CA grains may give higher interfacial areas per unit of volume. Mukhopadhyay et al. ${ }^{18}$ proposed in a recent work a method to make the interface of the CA grain smooth. It is doubtful that their method fully corrected for shape differences between CA and real polyhedral grains.

The present agreement between CA simulation and the experimental data provides two conclusions. The first is that the agreement validates our CA simulation methodology, showing that it is able to describe experimental data reasonably well, provided the necessary time and space dimensionalizations are correctly scaled, and any differences in grain shape are taken into account. The other conclusion is more subtle, and relates to our prior investigations on non-randomly located nuclei. In those investigations it was found that Equation 6, and to a lesser extent also Equation 10, gave an excellent fit even when nuclei were not located randomly.

This result confirmed Vandermeer's suggestion for adding more parameters or relationships to MPM methodology, such as, the contiguity, to assess deviations from random nuclei placement. Earlier works, such as that of V\&R did not measure contiguity. The absence of such measurements raises concern about previous analysis of experimental data carried out based on Equation 6, exclusively, and to a lesser extent analyses based on Equations 6 and 10. Such an analysis may yield good curve fitting, but will obtain fitting parameters that are inaccurate. For example, in previous works, Rios et al..$^{15,29,31}$ showed that CA simulations with non-randomly located nuclei yield a fitted value of the number of nuclei per unit of volume, $\mathrm{N}_{\mathrm{v}}$, and a time exponent for the velocity from Equation 6 that were different from the number of nuclei per unit of volume and time exponent used in the simulation. Consequently, in the present work, the fact that we reproduced experimental results using quantities extracted from analytical models validates the $\mathrm{CA}$ simulation and the analytical treatment by
V\&R. However, in studies in which one has less experimental data this could become an issue. This is particularly true in studies that report only the volume fraction as function of time data.

Thus, a methodology of analysis is proposed in this work coupling CA simulations and analytical description. This methodology can be explained as follows. Firstly, the data are analyzed using the analytical approach, Equations 6 and 10, that assumes randomness. From that starting point the interfacial velocity and the number of grains per unit of volume may be obtained. A CA simulation is then conducted using the same quantities with random nucleation. If the result differs from the experimental result it implies that the original assumption of randomness was not justified. On the other hand, if the CA simulation yields good agreement with experimental data, one is reassured that the random assumption was satisfied, and, consequently, the results from the analytical approach are correct. Of course, one might argue that many difficulties could be avoided by measuring the contiguity, or even the $\phi$ function. This is not always feasible. For example, in phase transformation studies one often uses indirect methods, e.g., dilatometry, which yield only $\mathrm{V}_{\mathrm{v}}$ vs. time curves. In other situations measurements of the interfacial area per unit of volume between transformed regions may be difficult, as the grain boundaries between transformed regions are not always easy to reveal.

Thus, CA simulation can be used to aid in the interpretation of microstructure evolution by simulating the process, and injecting conclusions, or even conjectures, inferred from analytical /experimental analysis of the data. This could be helpful in clarifying situations when the assumptions in which the analytical treatment is based are not met, as is the case of non-randomly located nuclei.

\section{Summary and Conclusions}

- Agreement was obtained between CA simulation with experimental data as well as with V\&R analytical description of the data of the recrystallization of an iron single crystal ${ }^{1}$;

- Proper time and space dimensionalization of CA simulation was required as well as consideration of the difference in shape between CA and experimental/spherical grains;

- The fact that experimental results were correctly reproduced using quantities extracted from analytical analysis not only validated the CA simulation but also validated the analytical treatment of V\&R; and

- A methodology of analysis is proposed in this work coupling CA and analytical description. A CA simulation using conclusions and parameters obtained from analytical treatment might or might not confirm the analytical results depending on the assumptions made in formulating the analytical treatment.

\section{Acknowledgments}

P. R. Rios is grateful to Conselho Nacional de Desenvolvimento Científico e Tecnológico, CNPq, and to Fundação de Amparo à Pesquisa do Estado do Rio de Janeiro, FAPERJ, for his financial support. T.C. Salazar and W. L. S. Assis are grateful to CNPq and CAPES, respectively, for their graduate scholarship. The authors are especially grateful to Professor Martin E. Glicksman for his careful reading of the manuscript.

\section{References}

1. Rios PR, Siciliano Jr F, Sandim HRZ, Plaut RL, Padilha AF. Nucleation and growth during recrystallization. Materials Research 2005; 8(3): 225-238.

2. Lauridsen EM, Poulsen HF, Nielsen SF, Jensen DJ. Recrystallization kinetics of individual bulk grains in $90 \%$ cold-rolled aluminum. Acta Materialia 2003; 51(15): 4423-4435. 
3. Vandermeer RA and Rath BB. Modeling recrystallization kinetics in a deformed iron single crystal. Metallurgical Transactions A 1989; 20A(3): 391-401.

4. Johnson WA, Mehl RF. Reaction kinetics in processes of nucleation and growth. Transactions AIME 1939; 135: 416-441.

5. Avrami MJ. Kinetics of phase change I general theory. The Journal of Chemical Physics 1939; 7(12): 1103-1112.

6. Avrami MJ. Kinetics of phase change II transformation-time relations for random distribution of nuclei. The Journal of Chemical Physics 1940; 8(2): 214-224.

7. Avrami MJ. Kinetics of phase change III granulation, phase change, and microstructure kinetics of phase change. The Journal of Chemical Physics 1941; 9(2): 177-184.

8. Kolmogorov NA. The statistics of crystal growth in metals. Isvestiia Academii Nauk SSSR - Seriia Matematicheskaia 1937; 1: 333-359.

9. Gokhale AM, Iswaran CV and DeHoff RT. Use of the stereological counting measurements in testing theories of growth-rates. Metallurgical Transactions A 1979; 10A(9): 1239-1245.

10. Gokhale AM. Use of stereological measurements for the study of grainboundary diffusion controlled precipitate growth-kinetics. Metallurgical Transactions A 1985; 16A(3): 456-457.

11. Gokhale AM. Application of microstructure modeling to the kinetics of proeutectoid ferrite transformation in hot-rolled microalloyed steels. Metallurgical Transactions A. 1986; 17A(9): 1625-1629.

12. De Hoff RT. In Hansen N, Jensen DJ, Leffers T, Ralph B, editors Annealing Processes-Recovery, Recrystallization and Grain Growth, Proceedings of the 7th Conference of Ris $\phi$ National Laboratory; 1986, Roskilde, Denmark. Roskilde: Risø National Laboratory. 1986; 35-52.

13. Vandermeer RA, Masumura RA, Rath BB. Microstructural paths of shapepreserved nucleation and growth transformations. Acta Metallurgica et Materialia. 1991; 39(3): 383-389.

14. Vandermeer RA. Microstructural descriptors and the effects of nuclei clustering on recrystallization path kinetics. Acta Materialia 2005; 53(5): 1449-1457.

15. Rios PR, Pereira LO, Oliveira FF, Assis WLS, Castro JA. Impingement function for nucleation on non-random sites. Acta Materialia 2007; 55(13): 4339-4348.

16. Hesselbarth HW, Göbel IR. Simulation of recrystallization by cellular automata. Acta Metallurgica et Materialia 1991; 39(9): 2135-2143.

17. Marx V, Reher FR, Gottstein G. Simulation of primary recrystallization using a modified three-dimensional cellular automaton. Acta Materialia 1999; 47(4): 1219-1230.

18. Mukhopadhyay P, Loeck M, Gottstein G. A cellular operator model for the simulation of static recrystallization. Acta Materialia 2007; 55(10): 551-564.

19. Raabe D. Introduction of a scalable three-dimensional cellular automaton with a probabilistic switching rule for the discrete mesoscale simula- tion of recrystallization phenomena. Philosophical Magazine A 1999; 79(10): 2339-2358

20. Raabe D. Cellular automata in materials science with particular reference to recrystallization simulation. Annual Review of Materials Research 2002; 32: 53-76.

21. Pezzee CF, Dunand DC. The impingement effect of an inert, immobile 2nd phase on the recrystallization of a matrix. Acta Metallurgica et Materialia 1994; 42(5): 1509-1524.

22. Sessa V, Fanfoni M, Tomellini M. Validity of Avrami's kinetics for random and nonrandom distributions of germs. Physical Review B 1996; 54(2): 836-841.

23. Goetz RL, Seetharaman V. Static recrystallization kinetics with homogeneous and heterogeneous nucleation using a cellular automata model Metallurgical and Materials Transactions A 1998; 29(9): 2307-2321.

24. Pineda E, Pradell T, Crespo D. Non-random nucleation and the Avrami kinetics. Philosophical Magazine A 2002; 82(1): 107-121.

25. Janssens KGF. Random grid, three-dimensional, space-time coupled cellular automata for the simulation of recrystallization and grain growth Modelling and Simulation in Materials Science and Engineering 2003; 11(2): 157-171

26. Dewri R, Chakraborti N. Simulating recrystallization through cellular automata and genetic algorithms. Modelling and Simulation in Materials Science and Engineering 2005; 13(2): 173-183.

27. Rios PR, Carvalho JJS, Salazar TC, Paula FVL, Castro JA. Cellular automata simulation of the effect of nuclei distribution on the recrystallization kinetics. Materials Science Forum 2004; 467-470: 659-664.

28. Rios PR, Oliveira JCPT, Oliveira VT, Castro JA. Comparison of analytical models with cellular automata simulation of recrystallization in two dimensions. Materials Research 2005; 8(3): 341-345.

29. Rios PR, Oliveira JCPT, Oliveira VT, Castro JA. Microstructural descriptors and cellular automata simulation of the effects of non-random nuclei location on recrystallization in two dimensions. Materials Research, 2006; 9(2): 165-170.

30. Rios PR, Pereira LO, Oliveira VT, Pereira MR, Castro JA. Cellular automata simulation of site-saturated and constant nucleation rate transformations in three dimensions. Materials Research 2006; 9(2): 223-230

31. Rios PR, Pereira LO, Assis WLS, Oliveira FF, Oliveira VT. Analysis of transformations nucleated on non-random sites simulated by cellular automata in three dimensions. Materials Research 2007; 10(2): 141-146.

32. Assis WLS. Investigação do efeito da nucleação, da velocidade de crescimento e da distribuição da energia armazenada na recristalização pelo método do autômato celular em três dimensões. [unpublished $\mathrm{M}$. Sc. thesis]. Volta Redonda: Universidade Federal Fluminense; 2006.

33. Cahn JW, Hagel W. Theory of the pearlite reaction. In: Zackay ZD, Aaaronson HI, editors. Decomposition of austenite by Diffusional Processes. New York: Interscience; 1960. 
